\title{
Aberrant Effective Connectivity of the Ventral Putamen in Boys With Attention-Deficit/Hyperactivity Disorder
}

\author{
Zhihui Lan ${ }^{1,2,3 *}$, Yunkai Sun ${ }^{1,2,3}$, Lei Zhao ${ }^{1,2,3}$, Yang Xiao ${ }^{1,2,3}$, Changxiao Kuai ${ }^{1,2,3}$, and Shao-Wei Xue ${ }^{1,2,3 凶}$ \\ ${ }^{1}$ Center for Cognition and Brain Disorders, the Affiliated Hospital, Hangzhou Normal University, Hangzhou, China \\ ${ }^{2}$ Institute of Psychological Science, Hangzhou Normal University, Hangzhou, China \\ ${ }^{3}$ Zhejiang Key Laboratory for Research in Assessment of Cognitive Impairments, Hangzhou, China
}

\begin{abstract}
Objective The connectivity alterations in the putamen were found in revealing the neural correlates of attention-deficit/hyperactivity disorder (ADHD), but whether the effective connectivity of the putamen is atypical in ADHD remains unclear. Investigating this abnormality contributes to describing the neural circuit of ADHD at the level of macrostructural organization.

Methods Data were acquired from thirty-two boys with ADHD and fifty-two matched typically developing controls (TDC) from Peking University (Peking) dataset deposited at the Neuroimaging Informatics Tools and Resources Clearinghouse (NITRC) platform. We examined the effective connectivity of the putamen using Granger causality analysis (GCA) and then determined whether these connections could differentiate ADHD from TDC.

Results Compared with TDC, the ADHD group showed decreased effective connectivity from the left ventral rostral putamen (VRP) to left calcarine (CAL), right medial part of the superior frontal gyrus, left orbital part of superior frontal gyrus and left middle occipital gyrus (MOG). Increased effective connectivity from the left inferior occipital gyrus and right lingual gyrus to left VRP was also found in ADHD. The result of the classification accuracy showed that $72.3 \%$ of participants were correctly classified using support vector machine. Moreover, GCA values from the left VRP to left CAL and left MOG were significantly correlated with hyper/impulsive scores of patients with ADHD.
\end{abstract}

Conclusion The findings may help extend our understanding of the ADHD-related neural loops.

Psychiatry Investig 2021;18(8):763-769

Key Words Attention-deficit/hyperactivity disorder; Effective connectivity; Granger causality analysis; Ventral putamen.

\section{INTRODUCTION}

Attention-deficit/hyperactivity disorder (ADHD) is a developmental and psychiatric disorder that commonly begins in childhood and may persist into adulthood. ADHD is more prevalent in males, characterized by inattention, impulsivity, and hyperactivity. With the high prevalence and large medical burden, many studies ${ }^{1-3}$ have sought to uncover the pathophysiology of ADHD. Several brain regions such as the putamen

\footnotetext{
Received: December 3, 2020 Revised: March 30, 2021

Accepted: June 3, 2021

$\triangle$ Correspondence: Shao-Wei Xue, $\mathrm{PhD}$

Center for Cognition and Brain Disorders, Hangzhou Normal University, No.2318, Yuhangtang Rd, 311121, Hangzhou, Zhejiang, China

Tel: +86-571-28867717, Fax: +86-571-28867717, E-mail: xuedrm@126.com

*These authors contributed equally to this work.

(c) This is an Open Access article distributed under the terms of the Creative Commons Attribution Non-Commercial License (https://creativecommons.org/licenses/by$\mathrm{nc} / 4.0$ ) which permits unrestricted non-commercial use, distribution, and reproduction in any medium, provided the original work is properly cited.
}

were found to be atypical in ADHD. ${ }^{4-6}$ The putamen is a neural hub which integrates projections from diverse cortical regions and relays to the thalamus and other cortex regions. Numerous studies have reported morphological and functional abnormalities in the putamen. For example, a previous structural magnetic resonance imaging (MRI) study found that participants with ADHD had significantly smaller volume in the putamen than healthy controls. ${ }^{7}$ A meta-analysis study has also documented the putamen abnormality in children with ADHD. ${ }^{8}$ Abnormal functional connectivity between the putamen and thalamic regions related to spatial working memory processing was observed in ADHD. ${ }^{9}$ A resting-state functional MRI (R-fMRI) study observed altered functional connectivity of the putamen in ADHD patients. ${ }^{10}$ These studies indicated that the alterations in the putamen may involve ADHD.

Granger causality analysis (GCA) is an approach to identify whether the current time series in a brain region or network (X) can be predicted by the past time series of another (Y). GCA 
can reveal the causal relationship between regions or networks by establishing the regression equation for X and Y. For example, using this method, a R-fMRI study found abnormal effective connectivity of the frontal cortex to cerebellum in ADHD patients compared with typically developing controls (TDC). ${ }^{11}$ Another study using GCA differentiated patients with ADHD from TDC based on effective connectivity among salience network and frontoparietal network. ${ }^{12}$ However, to the best of our knowledge, effective connectivity in the putamen in patients with ADHD remains unclear.

In the present study, our aim was to investigate the effective connectivity of the putamen using GCA. We first computed the fractional amplitude of low-frequency fluctuation (fALFF) in order to determine whether functional deficits in the putamen occurred, which can enhance the reliability of differences found in subsequent analysis. Converging evidence from fMRI studies ${ }^{13,14}$ has revealed localized abnormalities in some brain areas were associated with interregional functional dysconnectivity. Therefore, GCA of R-fMRI data was conducted to investigate the effective connectivity between the putamen and the rest of the brain regions in ADHD patients relative to TDC. We selected GCA values and fALFF values with significant betweengroup differences as features to differentiate patients with $\mathrm{ADHD}$ from TDC using a machine learning approach, which can evaluate the strength of relevant regions measures in distinguishing these two groups. Finally, we carried out a correlation analysis to identify the associations of the effective connectivity and fALFF values in the putamen with symptom severity underlying ADHD. We hypothesized that the effective connectivity in the putamen would be abnormal in ADHD compared with TDC and the effective connectivity with significant group difference would be correlated with symptom severity of ADHD.

\section{METHODS}

\section{Participants}

A total of thirty-two ADHD patients (age: $12.66 \pm 1.75$ years; gender: 32 males/0 female) and fifty-two age-matched righthanded TDC (age: 12.09 \pm 1.61 years; gender: 52 males $/ 0$ female) were included in the current study. These subjects were part of the Peking University (Peking) dataset archived at the Neuroimaging Informatics Tools and Resources Clearinghouse (NITRC) platform. ADHD participants were diagnosed based on the Diagnostic and Statistical Manual of Mental Disorders, Fourth Edition (DSM-IV) criteria. All data were obtained from the ADHD-200 Consortium (http://fcon_1000.projects.nitrc.org/indi/adhd200/).

Symptom severity of ADHD and TDC was assessed using Attention-Deficit/Hyperactivity Disorder Rating Scale (ADHD-RS). The exclusion criteria for the study included the fol- lowing: 1) participants who were right-handed males and age $>12$ years and $<16$ years were initially selected; 2 ) participants with intelligence quotient (IQ) greater than 80 were included according to the Wechsler Intelligence Scale for Chinese Children-Revised (WISCC-R); 3) participants with poor quality images or incomplete phenotypic data were excluded; 4) participants with excessive movement during R-fMRI scanning were excluded (translation $>2.5 \mathrm{~mm}$ or rotation $>2.5$ degrees in any direction). All study procedures were conducted in accordance with the Declaration of Helsinki and were approved by the local Institutional Review Board (IRB No. 20201124). Prior to participation, informed consent was received from all subjects and their parents/legal guardians.

\section{Imaging acquisition and preprocessing}

The images were acquired with a Siemens MAGNETOM Allegra syngo 3.0 T MR Scanner (Siemens AG, Medical Solutions, Erlangen, Germany). Functional images were collected by using a T2*-weighted gradient-recalled EPI sequence. A high-resolution T1-weighted structural image in the sagittal orientation was obtained with magnetization-prepared rapid gradient echo (MPRAGE) sequence. The detailed image acquisition parameters can be seen at the ADHD-200 website (http:// fcon_1000.projects. nitrc.org/indi/adhd200/).

Data processing was implemented using the Data Processing Assistant for Resting-State fMRI (DPARSF, http://www. rfmri.org/), Resting-State fMRI Data Analysis Toolkit (REST, http://www.restfmri.net/forum/REST) and custom code written in MATLAB. Image preprocessing consisted of: 1) removal of the first 10 functional images for the scan environmental adaptation; 2) slice timing correction on the remaining 226 slices; 3 ) performing head motion correction (translation $<2.5$ $\mathrm{mm}$ or rotation $<2.5$ degrees). Moreover, we performed twosample t-test to analyze the between-group differences of the 3 translational parameters (X, Y, and Z) and the 3 rotational parameters (yaw, pitch and roll). We did not observe any significant difference in the above 6 directions; 4) nuisance covariate regression (Friston-24 motion parameters, white matter signal and cerebrospinal fluid signal); 5) spatial normalization into Montreal Neurological Institute (MNI) space using the transformation derived from $\mathrm{T} 1$ segmentation, and resampling at $2 \times 2 \times 2 \mathrm{~mm}^{3}$; 6) spatial smoothing with a $4 \mathrm{~mm}$ full-width half-maximum isotropic Gaussian kernel; 7) temporal filtering (bandpass, 0.01-0.08 Hz).

\section{Voxel-wise fALFF and GCA analysis}

The DPARSF software was used to conduct fALFF analysis. The fast Fourier transform (FFT) was implemented to convert the time series of each voxel into a frequency domain to obtain the power spectrum. The square root of the power spectrum 
was integrated within a low-frequency band to obtain the amplitude of low-frequency fluctuation (ALFF) of each voxel. The fALFF reflects the relative contribution of the ALFF within a specific frequency band $(0.01-0.08 \mathrm{~Hz})$ to the ALFF of the entire frequency range. ${ }^{15}$ The fALFF is an effective measure to quantify intrinsic spontaneous neural activity across the whole brain.

We implemented GCA using REST software. The regressive models are as follows:

$$
\begin{aligned}
& \boldsymbol{Y}_{t}=\sum_{i=1}^{p} \boldsymbol{A}_{i} \boldsymbol{X}_{(t-i)}+\sum_{i=1}^{p} \boldsymbol{B}_{i} \boldsymbol{Y}_{(t-i)}+\boldsymbol{C Z}_{\boldsymbol{t}}+\boldsymbol{E}_{\boldsymbol{t}} \\
& \boldsymbol{X}_{t}=\sum_{i=1}^{p} \boldsymbol{A}_{i}^{\prime} \boldsymbol{Y}_{(t-i)}+\sum_{i=1}^{p} \boldsymbol{B}_{i}^{\prime} \boldsymbol{X}_{(t-i)}+\boldsymbol{C}^{\prime} \boldsymbol{Z}_{t}+\boldsymbol{E}_{t}^{\prime}
\end{aligned}
$$

In the above model, $Y_{t}$ and $X_{t}$ denote two time series, $A_{i}$ and $\mathrm{A}^{\prime}{ }_{\mathrm{i}}$ are signed-path coefficients, $\mathrm{B}_{\mathrm{i}}$ and $\mathrm{B}_{\mathrm{i}}{ }_{\mathrm{i}}$ are autoregression coefficients, $\mathrm{E}_{\mathrm{t}}$ and $\mathrm{E}_{\mathrm{t}}{ }_{\mathrm{t}}$ are residual, and $\mathrm{Z}_{\mathrm{t}}$ is covariates. The time series $\mathrm{X}_{t}$ significantly causes the time series $\mathrm{Y}_{\mathrm{t}}$ if the signed-path coefficient $A_{i}$ is significantly larger. Similarly, the $Y_{t}$ can be defined as a significant Granger cause to $X_{t}$ if the signed-path coefficient $\mathrm{A}_{\mathrm{i}}{ }_{\mathrm{i}}$ is significantly larger. In the present study, the time series for the left ventral rostral putamen (VRP) was defined as the seed time series $\mathrm{X}$, and the time series $\mathrm{Y}$ denoted the time series of all voxels in the brain. We estimated the $X$-to-Y effects and the Y-to-X effects. Finally, the GCA maps were Z-transformed for standardization.

\section{Support vector machine classification}

Based on the results of two-sample t-test, the significant between-group differences in the GCA values and fALFF values of the left VRP were selected as features for classification. The support vector machine (SVM) classifier with radial basis function was used to evaluate discrimination capability of ADHD from TDC. The accuracy was estimated by k-fold cross-validation $(\mathrm{CV})$ to evaluate the performance of this classifier.

\section{Statistical analysis}

We employed two-sample t-test to analyze the between-group differences in GCA values. Gaussian Random Field (GRF) theory was applied for cluster-level multiple comparisons correction (voxel level $\mathrm{p}<0.005$, cluster level $\mathrm{p}<0.05$ ). According to a previous study, multiple linear regression was conducted to control for the effect confounds, including age, verbal IQ, performance IQ, full IQ, and mean framewise displacement (FD). ${ }^{16}$ Pearson correlation analysis was carried out between the GCA values and AALFF values with ADHD symptom severity (inattentive scores and hyper/impulsive scores), while correcting for effects of age, verbal IQ, performance IQ, full IQ and mean
FD. The GCA values were extracted between the left VRP and the other regions that had significant $\mathrm{X}$-to-Y effects and/or Yto-X effects in the bivariate GCA.

\section{RESULTS}

\section{Demographic and clinical data}

The demographic characteristics are shown in Table 1. There were no significant differences in age $(\mathrm{p}=0.133)$ or mean FD $(\mathrm{p}=0.192$ ) between the ADHD and TDC groups. ADHD participants had significantly lower scores on verbal IQ $(p=0.001)$, performance IQ $(\mathrm{p}<0.001)$ and full IQ $(\mathrm{p}<0.001)$ than the TDC group. The inattentive scores $(\mathrm{p}<0.001)$ and hyper/impulsive scores $(\mathrm{p}<0.001)$ of the ADHD group were significantly higher than the TDC group.

\section{fALFF analysis}

Anatomically, the putamen is a highly heterogeneous structure consisting of three subregions in each hemisphere: the dorsal rostral putamen (DRP, peak MNI coordinates: $x= \pm 25$, $y=8, z=6$ ), dorsal caudal putamen (DCP, $x= \pm 28, y=1, z=3$ ), and $\operatorname{VRP}(\mathrm{x}= \pm 20, \mathrm{y}=12, \mathrm{z}=-3) .{ }^{17}$ These nuclei receive projections from several brain regions and are involved in various neural responses. We computed fALFF in order to determine whether functional deficits in the putamen occurred. The fALFF analysis was performed to obtain fALFF maps for each participant and then transformed to Z scores. Finally, the mean $\mathrm{Z}$ scores of fALFF were extracted from each spherical ROI respectively for subsequent analysis. Because the mean fALFF values in the left VRP ( $\mathrm{x}=-20, \mathrm{y}=12, \mathrm{z}=-3$, MNI coordinates) displayed significant between-group differences $(p<0.05$, false discovery rate corrected), and we did not find significant dif-

Table 1. Demographic and clinical data

\begin{tabular}{lccc}
\hline & $\begin{array}{c}\text { ADHD } \\
(\text { mean } \pm \text { SD })\end{array}$ & $\begin{array}{c}\text { TDC } \\
(\text { mean } \pm \text { SD) }\end{array}$ & p-value \\
\hline Number of subjects & 32 & 52 & \\
Gender (male/female) & $32 / 0$ & $52 / 0$ & \\
Age (years) & $12.66 \pm 1.75$ & $12.09 \pm 1.61$ & 0.133 \\
Handedness (R/L) & $32 / 0$ & $52 / 0$ & \\
Mean FD & $0.08 \pm 0.033$ & $0.07 \pm 0.027$ & 0.192 \\
Verbal IQ & $109.59 \pm 14.40$ & $120.23 \pm 14.30$ & 0.001 \\
Performance IQ & $95.78 \pm 13.82$ & $112.29 \pm 15.42$ & $<0.001^{*}$ \\
Full IQ & $103.72 \pm 10.62$ & $118.42 \pm 14.59$ & $<0.001^{*}$ \\
Inattentive scores & $27.81 \pm 4.05$ & $15.56 \pm 3.75$ & $<0.001^{*}$ \\
Hyper/impulsive scores & $21.66 \pm 5.88$ & $13.09 \pm 3.42$ & $<0.001^{*}$ \\
\hline
\end{tabular}

${ }^{*} \mathrm{p}<0.001$. ADHD, attention-deficit/hyperactivity disorder; TDC, typically developing controls; SD, standard deviation; R, right; L, left; FD, framewise displacement; IQ, intelligence quotient 
ference in the other five subregions, the left VRP was set as a region of interest ( $\mathrm{ROI}$ ) with a $3 \mathrm{~mm}$ radius referring to previous studies. ${ }^{18,19}$

Table 2. Altered effective connectivity from and to the left ventral rostral putamen in ADHD

\begin{tabular}{ccccc}
\hline $\begin{array}{c}\text { Brain } \\
\text { region }\end{array}$ & $\begin{array}{c}\text { Cluster size } \\
\text { (voxels) }\end{array}$ & $\begin{array}{c}\text { Peak } \\
\text { t-value }\end{array}$ & p-value & $\begin{array}{c}\text { Peak MNI (X, Y, Z) } \\
\text { coordinates }(\mathrm{mm})\end{array}$ \\
\hline
\end{tabular}

Effective connectivity from the left ventral rostral putamen

$\mathrm{ADHD}<\mathrm{TDC}$

$\begin{array}{lllll}\text { L CAL } & 173 & -4.24 & 0.01 & -4,-84,-10 \\ \text { R SFGmed } & 183 & -4.28 & 0.007 & 10,72,2 \\ \text { L ORBsup } & 212 & -4.48 & 0.003 & -20,70,-2 \\ \text { L MOG } & 159 & -3.92 & 0.017 & -38,-90,16\end{array}$

Effective connectivity to the left ventral rostral putamen

ADHD $>$ TDC

$\begin{array}{lrrrc}\text { L IOG } & 132 & 4.59 & 0.006 & -40,-72,-8 \\ \text { R LING } & 328 & 3.78 & <0.001 & 6,-74,-2\end{array}$

Significance level was set at voxel $\mathrm{p}<0.005$, cluster $\mathrm{p}<0.05$, GRF corrected. MNI, Montreal Neurological Institute coordinates; ADHD, attention-deficit/hyperactivity disorder; TDC, typically developing controls; R, right; L, left; CAL, calcarine; SFGmed, the medial part of the superior frontal gyrus; ORBsup, the orbital part of superior frontal gyrus; MOG, middle occipital gyrus; IOG, inferior occipital gyrus; LING, lingual gyrus; GRF, Gaussian Random Field

\section{Granger causality analysis}

The GCA revealed that ADHD patients showed significantly decreased effective connectivity from the left VRP to the left calcarine (CAL), right medial part of the superior frontal gyrus (mSFG), left orbital part of superior frontal gyrus (ORBsup), and left middle occipital gyrus (MOG) than TDC. ADHD patients exhibited increased effective connectivity from the left inferior occipital gyrus (IOG) and right lingual gyrus (LING) to the left VRP compared with the TDC group (Table 2 and Figure 1).

\section{Classification results}

We selected GCA values and AALFF values with significant between-group differences as classification features to differentiate patients with ADHD from TDC using SVM. We employed a 5-fold CV algorithm for the estimation of classification accuracy. After repeating this procedure 50 times, the mean classification accuracy reached $72.3 \%$ (range from 0.657 to $0.763, \mathrm{SD}=0.021$ ).

\section{Relationship between GCA values and fALFF values with clinical variables}

As shown in Figure 2A, the GCA values from the left VRP to left CAL were significantly positively correlated with the hyper/impulsive scores of ADHD patients $(r=0.39, p<0.05$, uncorrected). The GCA values from the left VRP to left MOG (Figure 2B) were negatively correlated with the hyper/impul-

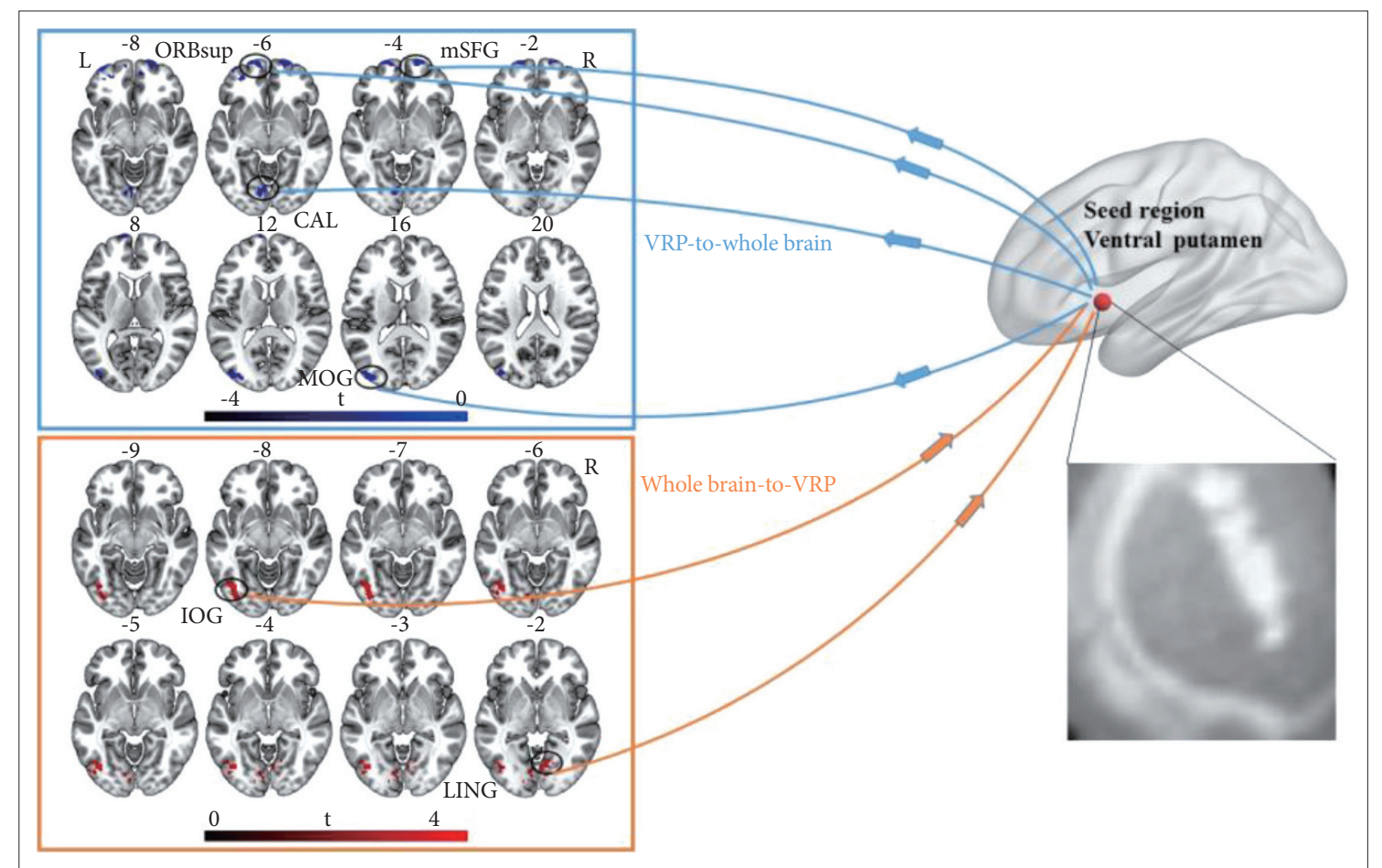

Figure 1. Significant differences in effective connectivity from and to the left VRP. Significance level was defined at voxel $p<0.005$, cluster p <0.05, GRF corrected. R, right; L, left; ORBsup, the orbital part of superior frontal gyrus, orbital; CAL, calcarine; mSFG, the medial part of the superior frontal gyrus; MOG, middle occipital gyrus; IOG, inferior occipital gyrus; LING, lingual gyrus; GRF, Gaussian Random Field. 

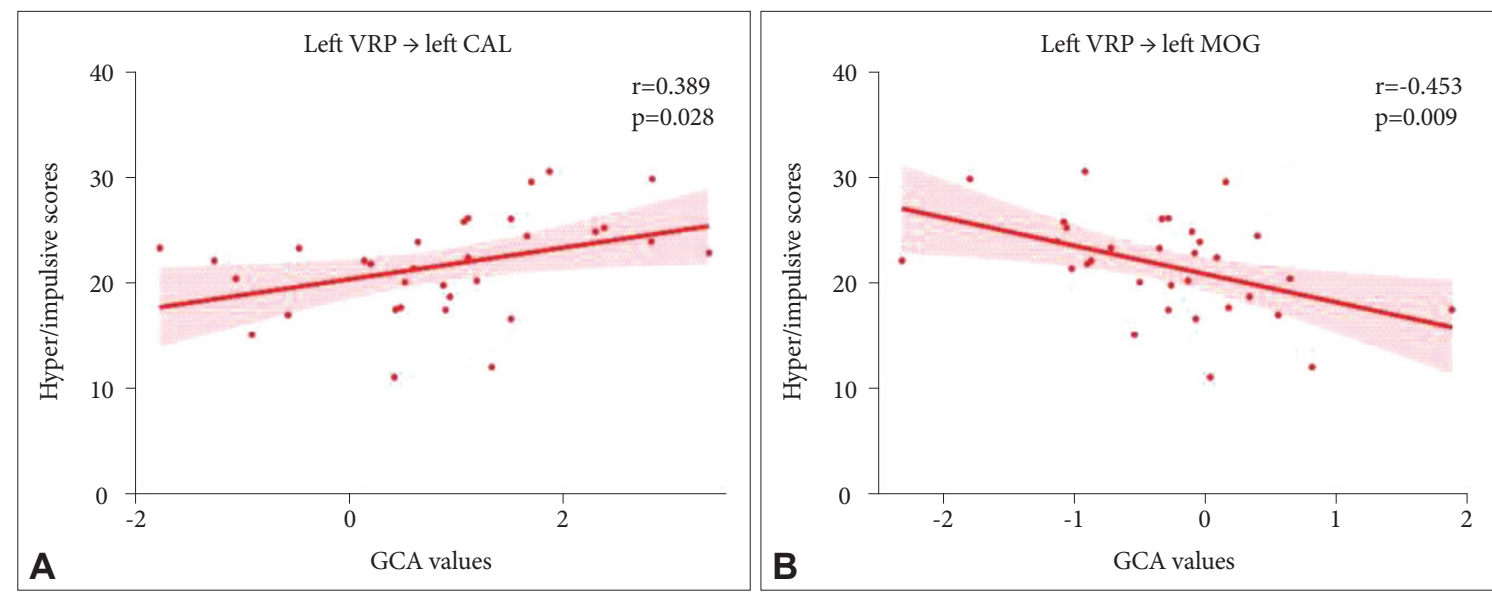

Figure 2. Correlations between GCA values and the hyper/impulsive scores in ADHD patients. A: Relationship between the GCA values from the left VRP to left CAL and the hyper/impulsive scores. B: Relationship between the GCA values from the left VRP to left MOG and the hyper/impulsive scores. VRP, ventral rostral putamen; CAL, calcarine; MOG, middle occipital gyrus; GCA, Granger causality analysis.

sive scores of $\mathrm{ADHD}$ patients ( $\mathrm{r}=-0.45, \mathrm{p}<0.05$, uncorrected). We did not observe any significant correlation between the GCA values of the left VRP and inattentive scores ( $p>0.05)$. Moreover, we did not find any significant correlation between the fALFF values of the left VRP and inattentive scores ( $p>0.05)$ and hyper/impulsive scores ( $\mathrm{p}>0.05)$.

\section{DISCUSSION}

In the present study, we aimed to identify whether the effective connectivity of the putamen was atypical in $\mathrm{ADHD}$, which was conducive to describing the neural circuit of ADHD at the level of macrostructural organization. We examined the abnormal information flow of the left VRP in ADHD group, which was more functionally specific. The GCA revealed that the effective connectivity of patients with ADHD from the left VRP to left CAL, right mSFG, left ORBsup and left MOG were decreased compared with TDC. However, the effective connectivity from the left IOG to left VRP and right LING to left VRP was enhanced in patients with ADHD. We selected GCA values and fALFF values with statistically significant between-group differences as classification features to identify ADHD patients from TDC using SVM. The classification results showed that the accuracy attained 72.3\%. Moreover, GCA values from the left VRP to left CAL were positively correlated with the hyper/ impulsive scores of ADHD patients, while GCA values from the left VRP to left MOG were negatively correlated with the hyper/impulsive scores of ADHD patients. The above findings indicated that abnormal effective connectivity in the putamen might be a valuable neuroimaging measure for ADHD.

The fALFF analysis was implemented to determine whether functional deficits in the putamen occurred among patients with ADHD. The results demonstrated that the left VRP existed significant group differences. Aberrant reward processing has been proven to play a crucial role in the pathophysiology underlying ADHD. ${ }^{20}$ The VRP is a key region in reward processing circuit, which receives projections from cortical areas (mainly the orbitofrontal cortex) and is mainly involved in anticipation of future rewards. ${ }^{21}$ A previous study considered that ADHD patients show symptoms of inattention or hyperactivity due to their inability to predict and evaluate future rewards. ${ }^{22}$ Therefore, the functional abnormalities in the VRP may be used as a potent predictor in ADHD diagnosis and treatment.

A previous R-fMRI study has demonstrated altered functional connectivity in the putamen among ADHD patients. ${ }^{9}$ However, this study provided only non-directional connectivity and the directed connectivity of putamen remain unclear. Our results showed reduced effective connectivity from the left VRP to bilateral superior frontal gyrus (SFG) in patients with ADHD compared with TDC. It is noted that the dysfunction of cortico-striato-thalamo-cortical (CSTC) circuit has been proposed as a prevailing hypothesis of pathophysiology of $\mathrm{ADHD}^{23-26}$ and the putamen is one of the main nuclei in striatum. Therefore, the abnormalities of CSTC circuit might result from decreased effective connectivity of the left VRP to bilateral SFG. These results are consistent with a previous study which reported patients with ADHD exhibited atypical functional connectivity between the left putamen and right SFG. ${ }^{10}$

Our results found that the effective connectivity of the left VRP to the left CAL and left MOG in patients with ADHD was reduced. Moreover, the effective connectivity of right LING to the left VRP and left IOG to the left VRP was significantly enhanced in patients with ADHD. These regions were mainly concentrated on the occipital gyrus, CAL and LING, which were involved in visual processing. In recent years, numerous neuroimaging studies have attached increasingly importance to investigate the dysfunction of frontal-striatal circuits in ADHD. ${ }^{27-30}$ However, some structural and functional MRI studies report- 
ed visual cortex abnormalities. For example, a previous R-fMRI study observed patients with ADHD exhibited increased regional homogeneity (ReHo) in the bilateral LING and occipital cortex. ${ }^{31}$ The LING is mainly involved in visual phonological processing ${ }^{32}$ and the hyper-activation in the LING might suggest that patients with ADHD need more attention resources to sustain visual phonological processing in daily life. A taskbased fMRI study also reported that ADHD patients displayed increased activation in the left LING relative to TDC..$^{33}$ As for the occipital cortex, abnormal activation in the occipital areas might result from a lack of inhibition of sensory perception. ${ }^{34}$ Furthermore, a structural MRI study reported that patients with ADHD had reduced volume of occipital gray matter. ${ }^{35}$ Another voxel-based morphometry study confirmed that patients with ADHD exhibited decreased whiter matter volume in the left CAL relative to TDC. ${ }^{36}$ The above results indicate that the abnormalities of the visual cortex are related to ADHD to some extent, thus, the visual cortex deserves deeper investigation to clarify its mechanism in patients with ADHD.

There were several limitations in the current study. Firstly, the current study was only limited to male participants due to $\mathrm{ADHD}$ is more prevalent in males, which might weaken the generalization of our results. Secondly, the small sample size might challenge the statistical power in our study. Further studies are needed to expand sample size. Thirdly, we observed visual cortex abnormalities, but the underlying causes of these alterations remain unclear. Therefore, further studies are required to determine whether and how these alterations reflect the neural mechanism in ADHD patients. Fourth, we have not performed a comprehensive assessment of the comorbidity of the data in the current study, which might bring challenges to the investigation and identification of ADHD. Accordingly, it is necessary to consider the psychiatric co-morbidity that is concurrent with it.

In summary, we identified the abnormal information flow of the left VRP in ADHD. Our study demonstrated alterations of effective connectivity between the left VRP with SFG and visual cortex, which may extend our understanding of the neural loop underlying ADHD.

\section{Availability of Data and Material}

The datasets generated or analyzed during the study are available from the corresponding author on reasonable request.

\section{Conflicts of Interest}

The authors have no potential conflicts of interest to disclose.

\section{Author Contributions}

Conceptualization: Shao-Wei Xue. Data curation: Zhihui Lan, Yunkai Sun. Formal analysis: Zhihui Lan. Funding acquisition: Shao-Wei Xue. Investigation: Zhihui Lan, Yunkai Sun. Methodology: Zhihui Lan, Lei Zhao. Project administration: Shao-Wei Xue. Resources: Shao-Wei Xue. Supervi- sion: Shao-Wei Xue. Validation: Shao-Wei Xue. Visualization: Yunkai Sun, Lei Zhao. Writing_original draft: Zhihui Lan, Yunkai Sun, Lei Zhao, Yang Xiao, Changxiao Kuai. Writing_-review \& editing: Shao-Wei Xue.

\section{ORCID iDs}

Zhihui Lan

Yunkai Sun

Lei Zhao

Yang Xiao

Changxiao Kuai

Shao-Wei Xue https://orcid.org/0000-0002-4891-4480 https://orcid.org/0000-0002-3716-0302 https://orcid.org/0000-0001-9348-3854 https://orcid.org/0000-0002-9746-9571 https://orcid.org/0000-0003-0943-2179 https://orcid.org/0000-0001-5441-4522

\section{Funding Statement}

This study was supported by the Natural Science Foundation of Zhejiang Province (LY17H180007), the Hangzhou Medical Key Discipline and the cultivation project of the province-leveled preponderant characteristic discipline in the College of Education (18JYXK036) of Hangzhou Normal University.

\section{REFERENCES}

1. Samea F, Soluki S, Nejati V, Zarei M, Cortese S, Eickhoff SB, et al. Brain alterations in children/adolescents with ADHD revisited: a neuroimaging meta-analysis of 96 structural and functional studies. Neurosci Biobehav Rev 2019;100:1-8.

2. Emond V, Joyal C, Poissant H. [Structural and functional neuroanatomy of attention-deficit hyperactivity disorder (ADHD)]. Encephale 2009;35:107-114.

3. Sun Y, Zhao L, Lan Z, Jia XZ, Xue SW. Differentiating boys with ADHD from those with typical development based on whole-brain functional connections using a machine learning approach. Neuropsychiatr Dis Treat 2020;16:691-702.

4. Luo X, Mao Q, Shi J, Wang X, Li CR. Putamen gray matter volumes in neuropsychiatric and neurodegenerative disorders. World J Psychiatry Ment Health Res 2019;3:1020.

5. Hoogman M, Bralten J, Hibar DP, Mennes M, Zwiers MP, Schweren LSJ, et al. Subcortical brain volume differences in participants with attention deficit hyperactivity disorder in children and adults: a crosssectional mega-analysis. Lancet Psychiatry 2017;4:310-319.

6. Rosch KS, Crocetti D, Hirabayashi K, Denckla MB, Mostofsky SH, Mahone EM. Reduced subcortical volumes among preschool-age girls and boys with ADHD. Psychiatry Res Neuroimaging 2018;271:67-74.

7. Qiu A, Crocetti D, Adler M, Mahone EM, Denckla MB, Miller MI, et al. Basal ganglia volume and shape in children with attention deficit hyperactivity disorder. Am J Psychiatry 2009;166:74-82.

8. Frodl T, Skokauskas N. Meta-analysis of structural MRI studies in children and adults with attention deficit hyperactivity disorder indicates treatment effects. Acta Psychiatr Scand 2012;125:114-126.

9. Mills KL, Bathula D, Dias TG, Iyer SP, Fenesy MC, Musser ED, et al. Altered cortico-striatal-thalamic connectivity in relation to spatial working memory capacity in children with ADHD. Front Psychiatry 2012; 3:2.

10. Cao X, Cao Q, Long X, Sun L, Sui M, Zhu C, et al. Abnormal restingstate functional connectivity patterns of the putamen in medicationnaive children with attention deficit hyperactivity disorder. Brain Res 2009;1303:195-206

11. Zhao D, Zheng S, Yang L, Tian Y. Causal connectivity abnormalities of regional homogeneity in children with attention deficit hyperactivity disorder: a rest-state fMRI study. ADMET DMPK 2017;5:242.

12. Cai W, Griffiths K, Korgaonkar MS, Williams LM, Menon V. Inhibition-related modulation of salience and frontoparietal networks predicts cognitive control ability and inattention symptoms in children with ADHD. Mol Psychiatry 2019 [Online ahead of print].

13. Wang X, Li F, Zheng H, Wang W, Zhang W, Liu Z, et al. Breakdown of the striatal-default mode network loop in schizophrenia. Schizophr 
Res 2015;168:366-372

14. Machner B, von der Gablentz J, Göttlich M, Heide W, Helmchen C, Sprenger A, et al. Behavioral deficits in left hemispatial neglect are related to a reduction of spontaneous neuronal activity in the right superior parietal lobule. Neuropsychologia 2020;138:107356.

15. Zou QH, Zhu CZ, Yang Y, Zuo XN, Long XY, Cao QJ, et al. An improved approach to detection of amplitude of low-frequency fluctuation (ALFF) for resting-state fMRI: fractional ALFF. J Neurosci Methods 2008;172:137-141.

16. Xu J, Wang H, Zhang L, Xu Z, Li T, Zhou Z, et al. Both hypo-connectivity and hyper-connectivity of the insular subregions associated with severity in children with autism spectrum disorders. Front Neurosci 2018;12:234

17. Di Martino A, Scheres A, Margulies DS, Kelly AM, Uddin LQ, Shehzad Z, et al. Functional connectivity of human striatum: a resting state FMRI study. Cereb Cortex 2008;18:2735-2747.

18. Di Martino A, Kelly C, Grzadzinski R, Zuo XN, Mennes M, Mairena MA, et al. Aberrant striatal functional connectivity in children with autism. Biol Psychiatry 2011;69:847-856.

19. Park J, Kim T, Kim M, Lee TY, Kwon JS. Functional connectivity of the striatum as a neural correlate of symptom severity in patient with obsessive-compulsive disorder. Psychiatry Investig 2020;17:87-95.

20. Sonuga-Barke EJ. Causal models of attention-deficit/hyperactivity disorder: from common simple deficits to multiple developmental pathways. Biol Psychiatry 2005;57:1231-1238.

21. O’Doherty J, Dayan P, Schultz J, Deichmann R, Friston K, Dolan RJ. Dissociable roles of ventral and dorsal striatum in instrumental conditioning. Science 2004; 304:452-454.

22. Nigg JT, Casey BJ. An integrative theory of attention-deficit/ hyperactivity disorder based on the cognitive and affective neurosciences. Dev Psychopathol 2005;17:785-806.

23. Gallo EF, Posner J. Moving towards causality in attention-deficit hyperactivity disorder: overview of neural and genetic mechanisms. Lancet Psychiatry 2016;3:555-567.

24. Zhu Y, Yang D, Ji W, Huang T, Xue L, Jiang X, et al. The relationship between neurocircuitry dysfunctions and attention deficit hyperactivity disorder: a review. Biomed Res Int 2016;2016:3821579.

25. Zhu Y, Jiang X, Ji W. The Mechanism of Cortico-Striato-Thalamo-Cor- tical Neurocircuitry in response inhibition and emotional responding in attention deficit hyperactivity disorder with comorbid disruptive behavior disorder. Neurosci Bull 2018;34:566-572.

26. Zhou M, Yang C, Bu X, Liang Y, Lin H, Hu X, et al. Abnormal functional network centrality in drug-naïve boys with attention-deficit/hyperactivity disorder. Eur Child Adolesc Psychiatry 2019;28:1321-1328.

27. Díaz-Heijtz R. [Biochemical bases and research in attention-deficit hyperactivity disorder]. Rev Neurol 2002;34(Suppl 1):S78-S81.

28. Krain AL, Castellanos FX. Brain development and ADHD. Clin Psychol Rev 2006;26:433-444.

29. Kelly AM, Margulies DS, Castellanos FX. Recent advances in structural and functional brain imaging studies of attention-deficit/hyperactivity disorder. Curr Psychiatry Rep 2007;9:401-407.

30. Swanson JM, Kinsbourne M, Nigg J, Lanphear B, Stefanatos GA, Volkow N, et al. Etiologic subtypes of attention-deficit/hyperactivity disorder: brain imaging, molecular genetic and environmental factors and the dopamine hypothesis. Neuropsychol Rev 2007;17:39-59.

31. Cao Q, Zang Y, Sun L, Sui M, Long X, Zou Q, et al. Abnormal neural activity in children with attention deficit hyperactivity disorder: a resting-state functional magnetic resonance imaging study. Neuroreport 2006;17:1033-1036.

32. Burton MW, Locasto PC, Krebs-Noble D, Gullapalli RP. A systematic investigation of the functional neuroanatomy of auditory and visual phonological processing. Neuroimage 2005;26:647-661.

33. Ko CH, Hsieh TJ, Wang PW, Lin WC, Chen CS, Yen JY. The altered brain activation of phonological working memory, dual tasking, and distraction among participants with adult ADHD and the effect of the MAOA polymorphism. J Atten Disord 2018;22:240-249.

34. Lou HC, Henriksen L, Bruhn P, Børner H, Nielsen JB. Striatal dysfunction in attention deficit and hyperkinetic disorder. Arch Neurol 1989;46:48-52.

35. Ahrendts J, Rüsch N, Wilke M, Philipsen A, Eickhoff SB, Glauche V, et al. Visual cortex abnormalities in adults with ADHD: a structural MRI study. World J Biol Psychiatry 2011;12:260-270.

36. Kumar U, Arya A, Agarwal V. Neural alterations in ADHD children as indicated by voxel-based cortical thickness and morphometry analysis. Brain Dev 2017;39:403-410. 